\title{
Research on the Impact of Chengdu-Chongqing High- Speed Railway on Tourism Economic Connection of Cities Along the Line
}

\author{
Li Liu $^{1 *}$ Lijuan Chen ${ }^{1}$
}

${ }^{1}$ School of Management, Chongqing University of Technology, Chongqing, China

*Corresponding author. Email: 1041067834@qq.com

\begin{abstract}
As an important part of the tourism industry, tourism transportation plays a vital role in the development of tourism. By constructing an accessibility model and a tourism economic connection model, the article compares and analyzes the changes in tourism transportation accessibility and tourism economic connections of the cities along the line before and after the opening of the high-speed rail. The results show that the high-speed rail shortens the time and space between cities along the line distance significantly improves the accessibility of tourist traffic in the areas along the route, but the change rate of tourist traffic accessibility is uneven in spatial distribution. The accessibility improvement rate of the first and last cities is the lowest, and the middle of the route, the city's accessibility improvement rate is relatively high; the intensity of tourism economic linkages in the cities along the high-speed rail line has generally increased, but due to the effect of distance attenuation, the growth rate of the tourism economic linkage strength between the site cities is not the same; the total amount of economic contacts has shown a growth trend of varying magnitudes, and its balance has improved.
\end{abstract}

\section{Keywords: Chengdu-Chongqing high-speed rail, accessibility, tourism economic connection}

\section{INTRODUCTION}

Tourism transportation is closely related to the development of tourism. As a link and bridge between tourist sources and destinations, tourism transportation has a profound impact on the attractiveness of tourism resources, the improvement of tourism service quality, and the development and planning of tourist destinations. On October 16, 2020, the Politburo meeting of the CPC Central Committee reviewed the "Plan for the Construction of the Chengdu-Chongqing Double-City Economic Circle". The meeting emphasized the promotion of the construction of the Chengdu-Chongqing Double-City Economic Circle and highlighted the synergistic and leading role of the two central cities of Chongqing and Chengdu. Chengdu-Chongqing area firmly establishes a game of chess thinking and integrated development concept, establishes a cooperation mechanism, sings the "Tale of Two Cities", handles the relationship between the center and the region, and strives to improve the development level and comprehensive competitiveness of the main city of Chongqing and Chengdu and promote the city. The development has changed from extensional expansion to connotation enhancement, with point-to-surface and balanced development, forming an integrated development metropolitan area with surrounding cities and counties [1]. Nowadays, my country's high-speed railway is developing rapidly. The high-speed railway transportation network has developed from a dense high-speed railway network of "four verticals and four horizontals" to "eight verticals and eight horizontals". High-speed railways rely on their advantages of high efficiency, speed, convenience, and safety. It has greatly improved the accessibility of tourism transportation, accelerated the flow of tourism elements in the areas along the route, and continued to optimize the structure of the tourism economic connection network. In recent years, with the continuous improvement of the high-speed rail network, analyzing the impact of high-speed rail on the accessibility of regions along the line has gradually become a hot issue in academic research, thereby providing decision-making support for the development of regional tourism.

Looking at the existing literature, there have been some research results on traffic accessibility in the academic community. Gutierrez $\mathbf{J}$ quantitatively analyzed the impact of the European Ossu Railway on the 
accessibility of the region along the line. The research results show that the high-speed rail can greatly improve the node cities in the region along the line. Traffic accessibility between [2]. According to Hiramats research, high-speed rail has a significant impact on the development of tourist destinations. Ahlfeldt, Gabriel M. and Feddersen, Arne believe that high-speed rail not only improves the accessibility of these areas, but also brings closer links between economic entities, but also makes the region's economy more accessible. Activities are more active [3]. Camille Kamga conducted an in-depth study on how high-speed rail can improve the quality and efficiency of tourism services, and made reasonable suggestions on how to use the high-speed rail system to promote urban integration [4]. Zhang Ming analyzed the rapid development of high-speed rail, its competition for regional tourism and transportation, and the changes in urban tourism and the spatial pattern of the tourist source market, and explained the impact of the development of high-speed rail on the tourism industry. [5]. Luo Pengfei used the three indicators of travel time, daily accessibility, and economic potential to conduct in-depth research on the changes in the accessibility of the Shanghai-Nanjing area under the influence of high-speed railways. The results prove that the economy of the areas along the line has not only been significantly changed, and the neighboring areas also benefited from this, and at the same time demonstrated the feasibility of the BeijingShanghai high-speed rail construction [6]. Wang Degen took the Beijing-Shanghai high-speed rail as an example to quantitatively study the impact of high-speed rail on regional traffic accessibility changes, and at the same time analyzed the high-speed rail effect and mechanism of the spatial structure of regional tourism flow [7]. Guo Jianke used a dual-core regional accessibility research system to deeply study the temporal and spatial evolution characteristics of comprehensive traffic accessibility in cities in Liaoning Province [8]. Taking the Harbin-Dalian high-speed rail as an example, Jiang Bo used the accessibility model and combined with the GIS inverse distance weighted interpolation method to analyze the dynamic changes of the accessibility of the cities along the line before and after the opening of the high-speed rail, and the change characteristics of the spatial pattern [9]. Li Yiman studied the temporal and spatial patterns of traffic accessibility in Zhejiang Province based on the weighted average travel time [10]. Jiang Haibing constructed an accessibility spatial analysis model based on the shortest path algorithm and network analysis technology, and measured the spatial pattern and changes of tourism accessibility before and after the planned highspeed rail opening to traffic in 2020 [11]. Mu Chenglin analyzed and studied the evolution of the tourism traffic pattern in the Yangtze River Delta under the background of the high-speed rail network [12]. To sum up, there are a lot of research results on accessibility in academia, but the research area is mainly concentrated in areas with more developed high-speed rail networks such as the
Beijing-Tianjin-Hebei, Pearl River Delta, and Yangtze River Delta, while less attention is paid to the western region. Therefore, this article Taking 11 station cities along the Chengdu-Chongqing area as the research object, with the support of GIS technology, through the construction of a tourism accessibility model and a tourism economic connection model, in-depth analysis of changes in the accessibility of tourism traffic before and after the opening of the Chengdu-Chongqing high-speed rail the spatial impact of tourism economic links between cities along the route provides reference and reference for the development of tourism in Chengdu-Chongqing region and high-speed rail tourism economy.

\section{RESEARCH AREA AND DATA SOURCES}

\section{A. Research area}

The Chengdu-Chongqing high-speed railway starts from Chengdu in the west and arrives in Chongqing in the east via Jianyang, Ziyang, Zizhong, Neijiang, Longchang, Rongchang, Dazu, Yongchuan and Bishan. The ChengduChongqing high-speed railway has a total length of 308 kilometers and started operation in December 2015, with a design maximum speed of $350 \mathrm{~km} / \mathrm{h}$. Through statistical calculations of the number of tourism resources and total tourist visits in the 11 station cities along the ChengduChongqing high-speed railway, it is found that the tourist resources of the cities along the route account for $40.8 \%$ of the total Sichuan-Chongqing area, and the total tourist visits account for $40.5 \%$ of the total. Moreover, the cities along the line are located in the core area of the ChengduChongqing area. The opening of the high-speed rail can improve the accessibility of the cities along the line and greatly shorten the time and space distance between the cities along the line. Therefore, the opening of the Chengdu-Chongqing high-speed rail will inevitably give the tourism industry in the Chengdu-Chongqing region. Development brings new vitality and growth poles, thus opening up a new situation for the development of tourism in Chengdu and Chongqing. In the area along the Chengdu-Chongqing high-speed rail, this study selected 11 stations in Chengdu, Jianyang, Ziyang, Zizhong, Neijiang, Longchang, Rongchang, Dazu, Yongchuan, Bishan, and Chongqing.

\section{B. Data sources}

Tourism economic data comes from the National Economic and Social Development Statistical Bulletin of these 11 districts and counties in 2013 and 2017. The average of the total number of tourist visits and total tourism revenue in 2013-2014 and 2016-2017 is used to measure tourism before and after the opening of the highspeed rail Main economic indicators of economic ties.

The original map data comes from the maps of Chongqing City and Sichuan Province published by China Map Publishing House in 2016, and the map data is 
vectorized using ArcGIS. Before the opening of the highspeed rail, the shortest travel mode between the various stations is road traffic. Using ArcGIS network analysis to calculate the shortest road distance between the various districts and counties, set the average speed to $80 \mathrm{~km} / \mathrm{h}$, so as to obtain the shortest travel time between the two cities before the opening of the high-speed rail. After the opening of the high-speed rail, based on the official website of the train ticket booking system 12306, the shortest travel time among the flights between the two cities is selected to determine the shortest travel time between the two cities.

\section{RESEARCH METHOD}

\section{A. Evaluation of accessibility of tourist transportation}

The accessibility of tourist traffic is the convenience of reaching a tourist destination from a certain location through the use of a specific transportation system [13]. In this paper, the weighted average travel time is used to evaluate the accessibility of tourism transportation in Chengdu-Chongqing area. This evaluation index can properly measure the time distance from the tourist source to the tourist destination, and it can also reflect the difference between the tourist source and the tourist destination. The economic level and transportation facilities, the lower the score of this indicator, the higher the level of accessibility of the city [14]. The specific formula is as follows:

$$
A_{i}=\frac{\sum_{j=1}^{n}\left(T_{i j} \times M_{j}\right)}{\sum_{j=1}^{n} M_{j}}
$$

In the formula, $\mathrm{Ai}$ is the weighted average travel time from city $j$ to city $i$ in the region, reflecting the accessibility of city $i$ in the region. The higher the index score, the lower the level of accessibility; $\mathrm{T}_{\mathrm{ij}}$ is the distance from city $\mathrm{j}$ to city $\mathrm{i}$ in the region $\mathrm{M}_{\mathrm{j}}$ is the quality of city $\mathrm{j}$ in the region, and the opening of high-speed rail has a greater impact on tourism. This article highlights the tourism function, $\mathrm{M}_{\mathrm{j}}=\sqrt{P_{j} V_{j}}$, among them, $P_{j} V_{j}$ is the geometric average of the total tourism revenue and total tourist arrivals of the cities along the route in 2013-2014 and 2016-2017; $n$ refers to the number of effective tourist sources.

\section{B. Tourism economic connection}

The tourism economic connection is modified according to the gravity model, and is an important indicator used to measure the strength of the tourism economic connection between cities in the region [15]. The specific formula is as follows:

$$
\begin{aligned}
& R_{i j}=\frac{\sqrt{P_{i} V_{i}} \sqrt{P_{j} V_{j}}}{T_{i j}^{2}} \\
& C_{i}=\sum_{j=1}^{n} R_{i j}
\end{aligned}
$$

In the formula, $\mathrm{Pi}$ and $\mathrm{Pj}$ represent the total tourist reception of city $\mathrm{i}$ and city $\mathrm{j}$ respectively; $\mathrm{Vi}$ and $\mathrm{Vj}$ represent the total tourism revenue of city $i$ and city $j$ respectively; $\mathrm{T}_{\mathrm{ij}}$ represents the shortest travel time from city $\mathrm{j}$ to city $\mathrm{i} ; \mathrm{R}_{\mathrm{ij}}$ is the amount of tourism economic connection, which reflects the degree and closeness of tourism economic connection between cities; $\mathrm{Ci}$ is the total tourism economic connection of city $i$, which reflects the status and role of city $i$ in the regional tourism economy.

\section{RESUlt ANALYSIS}

\section{A. Result analysis of urban tourist traffic accessibility}

Formula (I) is used to calculate the travel accessibility of 11 cities along the Chengdu-Chongqing high-speed rail before and after the operation ("Table I"), and use ArcGIS inverse distance weighted difference technology to simulate the spatial evolution pattern of travel accessibility of cities along the line ("Fig. 1"). It can be seen from Table I and Figure I that before the opening of the Chengdu-Chongqing high-speed rail, the weighted average travel time span of the 11 station cities along the line was [117.02-217.95], and the overall level of tourist traffic accessibility in the cities along the line was relatively low. Among them, Chengdu , Chongqing has the lowest level of tourist traffic accessibility, with a value exceeding 200 minutes. After the ChengduChongqing high-speed rail was opened to traffic, the overall level of accessibility of tourism and transportation in Chengdu-Chongqing area has been greatly improved, which significantly improved the accessibility of the 11 station cities along the line, and the span was reduced to [46.06-89.55], the weighted average travel time of cities along the route was reduced from 149 minutes before opening to traffic to 57 minutes, with a reduction rate of approximately $62 \%$, indicating that the difference in accessibility of tourism traffic between cities along the route is constantly decreasing, and the "2-hour tourism economy between counties and cities in the ChengduChongqing area" has been realized. ring". From the analysis of a single city, it is not difficult to find that except for Chongqing and Chengdu, the reduction rate of the other station cities is more than $60 \%$. According to the weighted average travel time reduction range from small to large, they are ranked as follows: Chengdu, Chongqing, Bishan, Yongchuan, Jianyang, Rongchang, Dazu, Longchang, Ziyang, Zizhong, Neijiang. It can be seen that Neijiang has the largest improvement in the accessibility of tourism traffic, while Chongqing and Chengdu have the smallest improvement in the accessibility of tourism traffic. That is to say, the accessibility of the stop cities (Neijiang, Zizhong, Ziyang) in the middle of the route has changed. The change rate of the accessibility of cities at the beginning and end of the high-speed rail line is relatively small. This shows that the space-time compression effect produced by the high-speed rail 
promotes the unbalanced spatial distribution of the change rate of the tourist traffic accessibility of the 11 station cities along the line. This is because before the opening of the high-speed rail, Chongqing and Chengdu were the core cities in the Sichuan-Chongqing region. Before the opening of the high-speed rail, their tourism economy was relatively strong, the transportation network system was relatively complete, and accessibility had been initially improved and improved. So after the opening of the high- speed rail to traffic, the degree of improvement in the accessibility of tourist traffic is not high. However, cities such as Neijiang, Zizhong, and Ziyang have not yet formed a sound transportation network system due to their limited tourism economic strength. Therefore, the opening of the high-speed rail significantly improves the accessibility of their tourism transportation and brings good opportunities for development.

TABLE I. THE WEIGHTED AVERAGE TRAVEL TIME OF CITIES ALONG THE CHENGDU-CHONGQING HIGH-SPEED RAIL BEFORE AND AFTER THE OPENING

\begin{tabular}{lllll}
\hline City & Before opening & After opening & Change amount & Decrease rate/\% \\
\hline Chengdu & 217.95 & 89.55 & -128.32 & -0.5891 \\
Jianyang & 147.02 & 57.20 & -89.82 & -0.6109 \\
Ziyang & 156.95 & 55.35 & -101.6 & -0.6473 \\
Zizhong & 146.81 & 50.23 & -96.58 & -0.6579 \\
Neijiang & 142.66 & 48.20 & -94.46 & -0.6621 \\
Longchang & 130.93 & 48.36 & -82.57 & -0.6306 \\
Rongchang & 128.60 & 48.86 & -79.74 & -0.6201 \\
Dazu & 129.31 & 48.64 & -80.67 & -0.6238 \\
Yongchuan & 117.02 & 46.06 & -70.96 & -0.6064 \\
Bishan & 119.14 & 47.34 & -62.79 & -0.6027 \\
Chongqing & 216.01 & 88.33 & -127.57 & -0.5911 \\
\hline
\end{tabular}
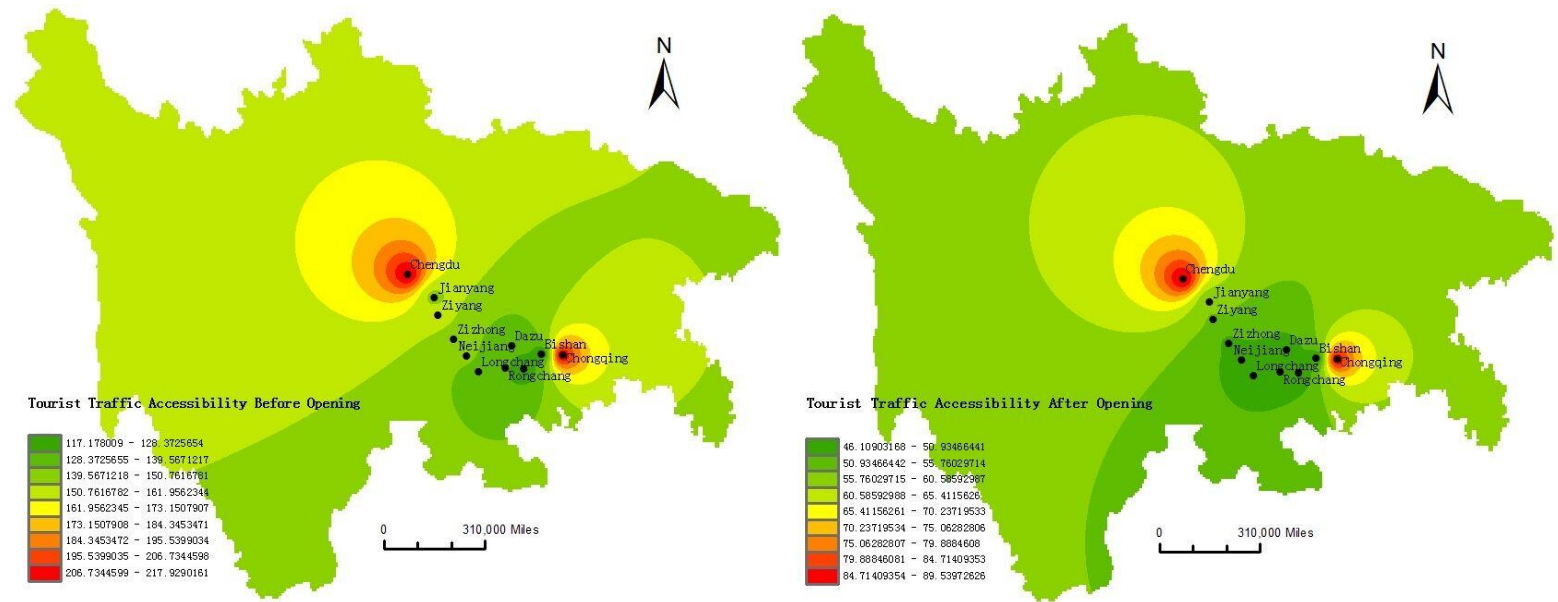

Fig. 1. Analysis on the spatial change of tourist traffic accessibility in cities along the route before and after the opening of high-speed rail.

\section{B. Analysis of changes in tourism economic connection}

Formula (II) is used to calculate the tourism economic linkage strength between the 11 station cities along the Chengdu-Chongqing high-speed rail before and after the high-speed rail is opened. The results are shown in "Table II". It can be seen from "Table II" that before the opening of the high-speed rail, there was a large difference in the intensity of tourism economic ties between the cities along the line, ranging from 0.27 to 690.77. The tourism economic ties between Chengdu and Jianyang, Ziyang, and Neijiang were relatively close, and the tourism economic ties were even above 100, the tourism economic link strength between
Chengdu and Zizhong, Longchang, and Rongchang is all lower than 100; the tourism economic link strength between Chongqing and Ziyang, Neijiang, Dazu, Yongchuan, and Bishan all exceeds 100, except for Jianyang and Ziyang tourism economy. Except for the strength of 135.39 , the strength of tourism economic ties between the other cities is less than 100, and the strength of tourism economic ties between many cities is even less than 10. It can be seen that Chengdu and Chongqing, as the central cities in the ChengduChongqing region, do not have a very obvious radiation effect on surrounding cities, and the main artery of tourism economic links between the site cities and the site cities has not yet formed. After the opening of the high-speed rail, the strength of the tourism economic 
ties between the cities along the route has increased significantly. Among them, the strength of the tourism economic ties between Chengdu and Jianyang, Ziyang, Zizhong, and Neijiang is as high as 1,000 or more, thus forming a close area of tourism economic ties. The most closely connected is the strength of the tourism economic ties between Chengdu and Ziyang reaching 5001.89. This is because The opening of the high-speed rail enables the interconnection between the two cities within 30 minutes, giving full play to the advantages of space proximity and highlighting the same city effect. The strength of the tourism economic connection between Chongqing and Bishan, Yongchuan, Dazu, and Neijiang is as high as 1,000 or more. This is mainly because apart from Neijiang, the distance between Chongqing and Yongchuan, Dazu, and Bishan is obvious, and as a central city, Chongqing has given full play to its radiating effect on surrounding cities. In the
55 pairs of cities composed of 11 station cities along the Chengdu-Chongqing high-speed rail line, with the exception of 16 pairs of cities with less than 100 tourism economic links, the remaining 10 pairs before opening to traffic increased to 39 pairs of cities after opening to traffic. The contact strength is greater than 100. It can be seen that with the improvement of the accessibility of tourism transportation, However, Rongchang has the weakest tourism economic link with Jianyang, which is only 9.79 , which is 913 times the highest value. In addition to the influence of location factors, Rongchang's tourism revenue and number of tourists are relatively low, and tourism income generation capacity is insufficient, leading to tourism reception weak ability. It can be seen that the intensity of tourism economic ties is directly related to the level of tourism development and relative geographic location of various regions.

TABLE II. THE TOURISM ECONOMY LINKAGE MATRIX OF CITIES ALONG THE CHENGDU-CHONGQING HIGH-SPEED RAILWAY BEFORE AND AFTER OPERATION

\begin{tabular}{|c|c|c|c|c|c|c|c|c|c|c|c|}
\hline & Chengdu & Jianyang & Ziyang & Zizhong & Neijiang & Longchang & Rongchang & Dazu & Yongchuan & Bishan & Chongqing \\
\hline Chengdu & & 4520.02 & 5001.89 & 1392.54 & 4222.33 & 608.62 & 183.05 & 612.64 & 523.75 & 242.65 & 8950.57 \\
\hline Jianyang & 282.34 & & 1348.54 & 85.66 & 252.82 & 31.92 & 9.79 & 36.54 & 30.84 & 18.36 & 501.94 \\
\hline Ziyang & 377.75 & 135.39 & & 612.59 & 1323.49 & 104.21 & 24.88 & 95.00 & 66.21 & 37.86 & 1148.60 \\
\hline Zizhong & 43.26 & 2.93 & 20.75 & & 1674.26 & 169.64 & 28.80 & 61.72 & 49.29 & 16.80 & 580.55 \\
\hline Neijiang & 128.48 & 8.93 & 39.88 & 68.29 & & 1582.52 & 167.94 & 406.54 & 373.14 & 138.75 & 3536.65 \\
\hline Longchang & 22.15 & 1.73 & 6.55 & 4.24 & 29.15 & & 144.01 & 324.16 & 207.64 & 68.72 & 844.74 \\
\hline Rongchang & 5.34 & 0.27 & 1.36 & 0.54 & 4.49 & 4.45 & & 222.89 & 163.90 & 34.17 & 319.58 \\
\hline Dazu & 23.75 & 2.22 & 5.08 & 2.04 & 11.98 & 6.85 & 1.68 & & 1041.20 & 259.67 & 1882.72 \\
\hline Yongchuan & 24.09 & 1.40 & 5.13 & 1.92 & 11.64 & 7.09 & 7.43 & 11.21 & & 581.72 & 3295.39 \\
\hline Bishan & 8.39 & 0.42 & 1.41 & 0.51 & 2.63 & 0.84 & 0.34 & 4.59 & 1.38 & & 3064.58 \\
\hline Chongqing & 690.77 & 34.28 & 108.16 & 29.00 & 161.31 & 67.67 & 21.09 & 181.04 & 466.65 & 198.05 & \\
\hline
\end{tabular}

a. Note: The lower left part is the data before activation, and the upper right part is the data after activation

TABLE III. THE TOTAL TOURISM ECONOMIC CONNECTION OF CITIES ALONG THE ROUTE BEFORE AND AFTER THE OPENING OF CHENGDUCHONGQING HIGH-SPEED RAIL

\begin{tabular}{cllll}
\hline City & Before opening & After opening & Change amount & Change rate/\% \\
\hline Chengdu & 1606.32 & 26258.06 & 24651.74 & 1534.67 \\
Jianyang & 469.91 & 6836.43 & 6366.52 & 1354.84 \\
Ziyang & 701.46 & 9763.27 & 9061.81 & 1291.85 \\
Zizhong & 173.48 & 4671.85 & 4498.37 & 2593.02 \\
Neijiang & 466.78 & 13678.44 & 13211.7 & 2830.38 \\
Longchang & 150.72 & 4086.18 & 3935.46 & 2611.11 \\
Rongchang & 46.99 & 1299.01 & 1252.02 & 2664.44 \\
Dazu & 250.44 & 4943.08 & 4692.64 & 1873.76 \\
Yongchuan & 537.94 & 6333.08 & 5795.14 & 1077.28 \\
Bishan & 218.56 & 4463.28 & 4244.72 & 1942.13 \\
Chongqing & 1958.02 & 24125.32 & 22167.3 & 1132.13 \\
\hline
\end{tabular}



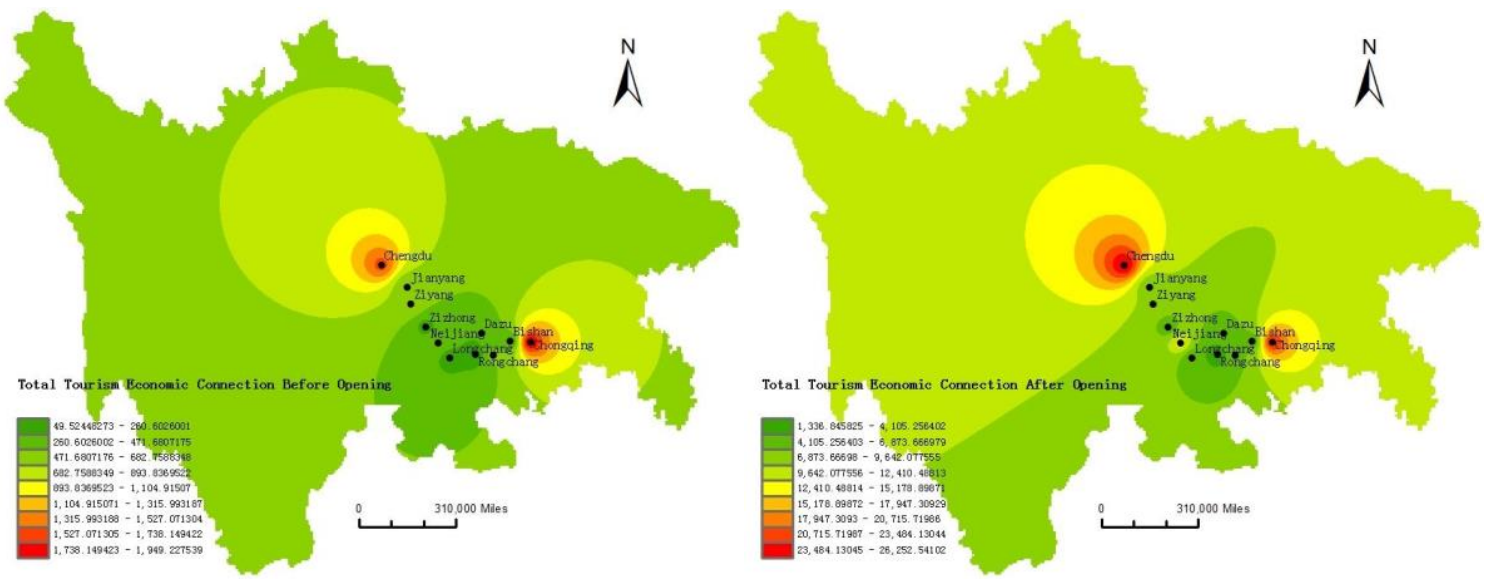

Fig. 2. Analysis of the spatial change of the total tourism economic connection of cities along the route before and after the opening of the high-speed rail.

\section{Analysis of the Total Tourism Economic Connection}

Formula (III) is used to calculate the total tourism economic connection of the 11 station cities along the Chengdu-Chongqing high-speed rail before and after the high-speed rail is opened. The results are shown in "Table III". It can be seen from "Table III" and "Fig. 2" that before the opening of the high-speed rail, the overall tourism economic linkages of the cities along the Chengdu-Chongqing high-speed rail were relatively low, except for Chengdu and Chongqing, which are relatively developed due to their relatively strong tourism economy. In the tourism transportation network system, its total tourism economic contacts are among the top, all above 1,000, followed by Ziyang and Yongchuan with more than 500 tourism economic contacts, followed by Zizhong, Longchang, Dazu, and Bishan. However, Rongchang's total tourism economic connection is the lowest, only 46.99. Although it is located in the middle of the Chengdu-Chongqing highspeed rail, the development potential of tourism resources is large, but the development of Rongchang's tourism industry is immature. The tourism industry is at a disadvantage. In general, the strength of regional tourism economic linkages along the ChengduChongqing high-speed rail line is centered on Chengdu and Chongqing, and radiates to the surrounding station cities. However, as the distance decreases, the overall trend is that the two ends are strong and the middle is weak. After the opening of the high-speed rail, the total tourism economic connection of the cities along the Chengdu-Chongqing high-speed rail has increased significantly. As central cities, Chengdu and Chongqing continue to maintain growth in the total tourism economic connection with the agglomeration effect, while the tourism economy of the peripheral cities It increases due to the diffusion effect. Its span range is [1299.01-26258.06], and the rate of change is between 10 times and 28 times. The smallest change in Yongchuan is 10 times, and the largest in Neijiang is 28 times. Because Neijiang is located in the middle of the Chengdu-Chongqing high-speed railway, it has a rich endowment of tourism resources. With the improvement of tourist traffic conditions between the tourist source markets, the accessibility of tourist traffic will increase, attracting potential tourists. The multiplier effect brought about by this makes the tourism economic connection multiplied. Overall, the Chengdu-Chongqing urban agglomeration has not undergone a qualitative change in the spatial pattern of tourism economic linkages. The status quo of Chengdu and Chongqing as tourism economic centers has not been weakened, and the situation of the peripheral cities is still not optimistic.

\section{CONCLUSION}

This paper takes 11 station cities along the Chengdu-Chongqing high-speed rail as the research object. Based on the accessibility model and the tourism economic connection model, this paper compares and analyzes the changes in the tourism transportation accessibility and tourism economic connection strength of the cities along the high-speed rail before and after the opening of the high-speed rail. And draw the following conclusions:

- The high-speed rail shortens the time and space distance between the cities along the line, and significantly improves and improves the accessibility of tourism transportation in the areas along the line. However, the change rate of the accessibility of tourism transportation is uneven in spatial distribution, thus presenting the first-end city. The accessibility change rate is relatively small, and the accessibility change rate of cities in the middle section of the line such as Zizhong, Ziyang, Neijiang, Rongchang, and Dazu is relatively large. 
- The intensity of tourism economic ties in the cities along the high-speed rail line has generally increased, but due to the effect of distance attenuation, the growth rate of the intensity of tourism economic ties between the station cities is not the same. At the same time, the opening of the high-speed rail has enhanced the spatial diffusion effect of Chengdu and Chongqing as central cities, and has greatly improved the radiation and driving role of the surrounding station cities such as Ziyang, Neijiang, Dazu, and Yongchuan. Neighboring cities should seize this development opportunity, develop new tourism products such as day trips and surrounding tours, strengthen and improve infrastructure construction, so as to improve their tourism competitiveness.

- The total tourism economic connections of the cities along the high-speed rail show a growth trend of varying degrees, and the balance has improved, but the overall tourism spatial pattern has not undergone substantial changes, and the situation of peripheral cities is still not optimistic.

\section{References}

[1] The Political Bureau of the CPC Central Committee held a meeting to deliberate on the "Plan for the Construction of the Chengdu-Chongqing Double-city Economic Circle", Xi Jinping, General Secretary of the CPC Central Committee, chaired the meeting. Xinhuanet. 2020-10-16.

[2] Gutierrez J, Gonzalez R, Gomez G The European high-speed train network:Predicted effects on accessibility Patterns[J]. 1996, 4(4): 227-238.

[3] Hiramatsu T. Unequal regional impacts of high speed rail on the tourism industry: a simulation analysis of the effects of Kyushu Shinkansen [J]. Transportation, 2018, 45(2):677-701.

[4] Camille Kamga. Emerging Travel Trends, high speed rail and the Public Reinvention of U.S. Transportation[J].Transportation Policy, 2015,37(11).

[5] Zhang Ming. The expected impact of high-speed railways on my country's tourism industry and countermeasures $[\mathrm{J}]$. Value Engineering, 2010, 29 (11): 227-228.

[6] Luo Pengfei, Xu Yilun, Zhang Nannan. Research on the impact of high-speed railway on regional accessibility: Taking the Shanghai-Nanjing area as an example [J]. Economic Geography, 2004(03): 407-411.

[7] Wang Degen, Chen Tian, Lu Lin, etc. The effect and mechanism of high-speed rail on the spatial structure of regional tourism flows: A case study of China's Beijing-Shanghai highspeed rail[J]. Acta Geographica Sinica, 2015, 70(2): 214-233.

[8] Guo Jianke, Wang Dandan. Research on dual-core regional traffic accessibility based on internal and external connections: Taking Liaoning Province as an example [J]. Economic Geography, 2015-11, 35(11): 72-84.

[9] Jiang Bo, Chu Nanchen, Wang Yuan, Yu Xiaolei, Zhao Yinghui, Xue Rui. Urban accessibility measurement under the influence of high-speed railway and simulation analysis of its spatial pattern: taking Harbin-Dalian high-speed railway as an example[J]. Economic Geography, 2014: 58-62.
[10] Li Yiman, Xiu Chunliang. Research on the Spatio-temporal Pattern of Traffic Accessibility in Zhejiang Province Based on Weighted Average Travel Time[J]. Human Geography, 201404, (4): 155-159.

[11] Jiang Haibing, Liu Jianguo, Jiang Jinliang. Research on the accessibility of national tourist attractions under the influence of high-speed railways[J]. Tourism Tribune, 2014, 29(7): 58-67.

[12] Mu Chenglin, Lu Lin, Huang Jianfeng. Research on the pattern and connection of tourism traffic in the Yangtze River Delta under the high-speed rail network $[\mathrm{J}]$. Economic Geography, 2015, 35 (12): 193-202.

[13] Ji Xiaofeng, Hao Jingjing, Chen Fang. Comprehensive transportation accessibility and spatial differentiation and coupling of logistics economy[J]. Transportation System Engineering and Information, 2015, 15(5): 24-31.

[14] Guo Jianke, Wang Shaobo, Wang Hui, et al. Research on the spatial effect of Harbin-Dalian high-speed rail on the tourism supply and demand market of northeastern cities: analysis based on the accessibility of scenic spots[J]. Advances in Geographical Sciences, 2016, 35(4): 505 -514.

[15] Chen Bowen, Lu Yuqi. The spatial distribution relationship between traffic accessibility and economic activities: Taking Jiangsu Province as an example [J]. Economic Geography, 20101, 36(1): 62-68. 